\title{
Politiques éducatives et analyse des inégalités: de quelques vertus heuristiques des comparaisons franco-britanniques
}

Marie Duru-Bellat

$\mathrm{D}$ ans l'analyse des inégalités à l'école comme dans les autres domaines des sciences sociales, la perspective comparative est toujours heuristique. En l'occurrence, les comparaisons internationales introduisent, quand on s'attache à comprendre un phénomène donné, de nouvelles sources de différenciation qui rendent visibles un certain nombre de relations et de processus qu'un point de vue purement local interdit d'appréhender. Outre cette ouverture quant aux facteurs pris en compte, les comparaisons aident à prendre conscience des impensés, notamment idéologiques, qui imprègnent le travail du sociologue lui-même, notamment par rapport à la question "chargée » des inégalités à l'école (Duru-Bellat, 1999).

Dans ce texte, il ne s'agit pas d'engager une nouvelle (et systématique) comparaison FranceAngleterre, mais plus simplement d'illustrer, par quelques exemples, en quoi les comparaisons franco-britanniques, dont J.-C. Forquin fut, en France, un pionnier, sont effectivement très stimulantes, et ce en pointant quelques registres où la comparaison bouscule la façon de penser les inégalités et les politiques éducatives, et aussi d'analyser les inégalités de carrières scolaires.

\section{DÉFINIR ET INTERPRÉTER LES INÉGALITÉS, CONCEVOIR ET JUSTIFIER LES POLITIQUES ÉDUCATIVES}

La problématique britannique de l'égalité des chances est a priori assez proche de la conception française : l'école est censée être accessible à tous ceux qui sont capables d'y réussir, quels que soient leur origine sociale et leur sexe. Mais derrière cette toile de fond méritocratique, il y a, plus fortement affirmée en Grande-Bretagne, une longue tradition d'une éducation différenciée selon les aptitudes et les classes sociales, alors qu'en France, l'égalité a été, jusqu'à une date récente, identifiée au fait d'offrir à tous des prestations strictement identiques. En GrandeBretagne, la méritocratie n'apparaît donc pas contradictoire, différence notable avec la France, avec une différenciation de l'offre...

L'évolution même des débats autour de l'égalité des chances scolaires (ainsi d'ailleurs que la place qu'y ont prise les sociologues) présente également des points communs et des différences, en France et en Grande-Bretagne. Dans la première moitié $d u X^{e}$ siècle, des chercheurs comme Burt soutiennent que l'intelligence est héréditaire et varie selon les groupes sociaux. 
Cette perspective a sensibilisé au problème des décalages inévitables entre milieu social et intelligence (les «bien doués" des milieux populaires...). On retrouve ces thèmes en France au début du $X X^{e}$ siècle avec le développement de la psychologie appliquée et de l'orientation. Mais la volonté progressiste d'orienter sur la seule base des aptitudes n'aura pas d'incidences institutionnelles majeures, car elle va avant tout concerner la frange d'élèves que leur origine sociale écarte des études générales longues. En GrandeBretagne, ce souci méritocratique - faire en sorte que seules les " aptitudes " commandent les carrières scolaires - aura des conséquences éducatives plus marquées et plus précoces. Ainsi, dès la première moitié du siècle, les plus "méritants" pourront dans certains cas, sur la base de la réussite à un concours (et l'utilisation de tests paraît alors évidente), être dispensés de frais pour une scolarité en Grammar School.

À partir des années 50-60, les débats autour de la notion d'aptitude se font très vifs. Les premiers sociologues de l'éducation britanniques se sont positionnés d'emblée en refusant l'hérédité : le "marché » du sociologue de l'éducation, c'est la mise à jour des "déterminants sociaux de l'éducabilité ". On peut les rapprocher en cela des sociologues français des années 60 (cf., à propos de l'origine innée des échecs scolaires, le « il vaut mieux douter trop que trop peu » de Bourdieu et Passeron). La France suivra en effet assez fidèlement cette évolution, mais avec un décalage temporel.

Comme dans les autres pays européens, le thème monte, tout au long du $X X^{e}$ siècle, selon lequel il serait coupable et dangereux, dans un contexte de concurrence économique, de ne pas exploiter toutes les "ressources de talents " de la nation: l'expression "pool of ability " est alors très fréquente. Exploiter l'intégralité du «capital humain " apparaît comme un gage à la fois de richesse économique et d'intégration sociale. Ce dernier thème (l'intégration sociale) est mis en avant dans les deux pays, où l'on compte sur la portée intégratrice d'une scolarisation/socialisation commune. On va donc s'efforcer de lever les barrières institutionnelles ou financières auxquelles se heurtent les enfants des milieux populaires, et pour ce faire on s'engage dans un développement de l'offre de places gratuites.

Mais en Grande-Bretagne, quand I'Education Act de 1944 rend le secondaire gratuit et acces- sible à tous, il donne des responsabilités au niveau local par souci d'une adaptation aux besoins de tous ("local needs"). Le souci d'une certaine justice sociale n'a donc jamais coïncidé avec la définition d'une formation uniformément offerte à tous comme en France. On est en effet persuadé que seules des filières diversifiées peuvent répondre à la diversité des aptitudes: on accède soit à une Grammar, soit à une Secondary Modern, soit à une Secondary Technical, sur la base d'un examen (le « eleven + "). En même temps, comme en France, on a eu tendance à considérer comme évident que démocratiser l'accès à l'éducation, c'était permettre à tous les enfants d'accéder à ce qu'on considérait comme l'archétype de l'éducation: Ia Grammar pour tous comme la culture du lycée pour tous... Avec donc, dans les deux pays, une tension entre égalité d'offre d'un modèle archétypique, et différenciation, différenciation souhaitable (pour les Anglais)... ou de fait inévitable, mais non sans mauvaise conscience (pour les Français).

Le système traditionnel anglais a été remis en cause du fait de critiques venant d'horizons multiples (mais auxquelles les sociologues ont participé activement). Tout d'abord, on dénonce le bien-fondé d'une sélection aussi précoce. Par ailleurs, se répand l'idée selon laquelle, dans les inégalités sociales d'éducation, les différences interindividuelles ne sont pas seules en cause et que l'école elle-même, de par son organisation, a une part de responsabilité. On pense en particulier aux travaux sur le streaming, très illustratifs de cette capacité des sociologues britanniques à s'emparer de problèmes très concrets du système éducatif, le mode de groupement des élèves en l'occurrence, pour en faire un sujet de recherche aux incidences théoriques non négligeables, avec tout ce qui concerne les processus d'étiquetage notamment. Ceci amène à souligner qu'en GrandeBretagne l'articulation entre politiques éducatives et recherche a été précoce, dès l'après-guerre, et forte: les sociologues ont été financés par les politiques pour instruire, sur la base d'enquêtes quantitatives, la question des inégalités sociales à l'école (ce qu'on a appelé "l'arithmétique politique »). En France, l'enquête de I'INED (conduite par les sociologues Girard et Bastide et mettant pour la première fois en pleine lumière l'ampleur des inégalités sociales à l'école) a été citée dans un certain nombre de textes officiels, mais l'articulation semble néanmoins bien moins forte. 
Toujours est-il qu'en Grande-Bretagne, pour contrer ces inégalités sociales véhiculées par la différenciation précoce du système, des écoles " compréhensives » sont mises en place dès 1965, très progressivement: $1 \%$ des élèves en 1951, $5 \%$ en 1961, $35 \%$ en 1971, $90 \%$ en 1981. Parallèlement, face aux inégalités sociales de réussite qui subsistent au sein des nouvelles filières unifiées, les sociologues ont importé des États-Unis le concept de "cultural deprivation", et l'éducation compensatoire s'est fortement développée dès les années 60 . Plus tard, avec la Nouvelle Sociologie de l'Éducation, les sociologues analyseront ces inégalités non plus comme une résultante du milieu social de l'élève mais bien comme une production du système éducatif luimême, qui construit une conception du savoir et de l'intelligence qui discrimine certains enfants. La perspective change donc de manière radicale: l'école n'est plus considérée comme une force de progrès, mais comme "une partie du problème ", puisqu'elle impose une culture dominante pour reproduire une société de classe. Ce renouvellement de perspective s'inscrit dans un changement de climat politique. D'un côté, on est passé de la confiance en la science du courant de l'arithmétique politique, au scepticisme de la Nouvelle Sociologie de l'Éducation, de l'autre, de la confiance en l'école comme vecteur d'intégration à une vision disons moins positive de son rôle.

Depuis les années 1980, on assiste en GrandeBretagne à une montée des attaques de la droite contre à la fois la gauche et la sociologie accusées d'être à l'origine de la "baisse de niveau " supposée. Parallèlement, deux thèmes font une percée remarquable. Tout d'abord, la nécessité de maintenir (mieux, d'élever) les "standards" académiques, et de développer l'évaluation des élèves; c'est un des objectifs essentiels que poursuit l'instauration d'un Curriculum National. Les responsabilités d'un État qui se veut "modeste " consistent à définir les "standards " et à en contrôler la réalisation. Même si le souci d'une évaluation standardisée se développe également en France, le système anglais reste marqué par le souci de mesurer au plus près la diversité des compétences des élèves (avec le système de certification par niveaux hiérarchisés, dans les matières de son choix). Le fait que tous les élèves (et non plus seulement ceux qui le souhaitaient ou étaient encouragés à le faire, comme auparavant) puissent voir leurs compétences certifiées [ce qu'ont voulu les réformes engagées en 1984, notamment la création du GCSE (1)] est également perçu en Grande Bretagne comme une étape dans la démocratisation du système.

Par ailleurs, le thème du choix de l'école fait une percée très forte dans les années 80 , avec le retour au pouvoir des conservateurs. Depuis les "black papers » publiés par la droite entre 1969 et 1977, qui commencent à pénétrer le milieu éducatif au début des années 80 , on assiste à une montée de la volonté de rogner les pouvoirs de l'État et de s'en remettre au marché et aux bienfaits de la compétition. La notion de choix est mise en avant systématiquement à partir du milieu des années 80. Mais si, dans l'Education Act de 1988, l'accent est mis sur le choix et le marché, dans le même temps, on instaure un curriculum national, comme si l'État avait à spécifier les conditions dans lesquelles cette compétition doit prendre place, en définissant ce que l'école doit apprendre, au moins à titre minimum.

Mais le thème du choix n'a pas une connotation idéologique parfaitement univoque (comme on aimerait pouvoir le croire en France). II reflète également la préoccupation «traditionnelle » des Britanniques pour une diversification de l'éducation en fonction des aptitudes et des besoins de l'enfant. Le processus de "comprehensivization" a laissé en suspens cette question, qui préoccupe à la fois la "gauche " et la "droite "; une certaine diversification des écoles était d'ailleurs soutenue, au moins initialement, par une partie des enseignants de gauche, comme susceptible de permettre une meilleure adaptation aux besoins des enfants (Walford, 1994).

Si un parallèle s'impose avec l'assouplissement de la carte scolaire initié au début des années 80 en France, il est clair que la politique suivie en Grande-Bretagne va beaucoup plus loin, notamment à partir de l'Education Act de 1988. La thématique du choix y est fortement affirmée, à travers l'introduction du recrutement libre des écoles, dont le financement devient largement dépendant du nombre d'élèves qu'elles réussissent à attirer. Soit les écoles peu choisies amélioreront leurs performances, soit elles fermeront. Dans un contexte de chute des effectifs, le financement des écoles et donc leur survie vont découler largement des décisions individuelles des parents. L'arrivée au pouvoir des travaillistes en 1997 ne semble pas devoir modifier l'accent 
qui est mis depuis les années 80 sur le choix des parents et la compétition entre écoles pour des performances accrues (Gibson et Asthana, 1999). La priorité reste, selon les déclarations politiques de 1997, "les standards, non les structures".

Les valeurs qui sous-tendent la confiance dans un mode de fonctionnement régi par le marché et non piloté d'en haut semblent donc bien ancrées. Du côté des usagers, il est clair que le système de choix présuppose un ensemble de valeurs qui donne la primauté à la comparaison, à la compétition individuelle, à la mobilité, au planning à long terme, au détriment des valeurs d'intégration dans la communauté locale. Mais la conviction du bien fondé d'une offre d'éducation différenciée est tellement prégnante qu'on est prêt à admettre une certaine sélection si elle se présente comme une spécialisation (comme le montre l'exemple des City Technogy Colleges). Mais s'agit-il de fait d'une demande de formations différenciées ou tout simplement d'une demande de formations sélectives? Aux yeux des parents, les écoles spécialisées ou à statut spécial mises en œuvre en Grande-Bretagne dans les années 80 ont été plutôt perçues comme des écoles sélectives, comme une extension de la formation traditionnelle d'élite («la Grammar pour tous»...). En Grande-Bretagne comme en France, toute différenciation tend à être interprétée comme une hiérarchisation.

Notons, en passant, la concomitance, en France comme en Grande-Bretagne, entre ces grands infléchissements des politiques éducatives et de grands tournants démographiques. Le développement des écoles "compréhensives" (ou du collège) coïncide avec une forte extension des populations scolaires; la solution la plus simple pour faire face à cette " explosion scolaire ", c'est évidemment la structure unique. À l'inverse, la thématique du choix coïncide avec un repli démographique. C'est très net en Grande-Bretagne : alors que dans l'après-guerre, le souci dominant était de trouver une place pour chaque enfant (régnait alors plutôt une pénurie de places), on assiste à partir de 1985 à une baisse du nombre d'enfants à scolariser (-30\% jusqu'en 1987). À partir des années 70 , beaucoup d'écoles ont de la place, ce qui rend plus difficile de s'opposer aux demandes de choix des familles.

Un mot sur un autre aspect du fonctionnement du système anglais qui bouscule quelque peu certains schémas bien ancrés en France, celui de l'autonomie des établissements. Elle est, on le sait, nettement plus consistante en Grande-Bretagne (on parle de "local management of schools", ou de "self-managing school concept»), où elle est censée permettre à l'établissement d'être à l'écoute des besoins de la communauté; l'établissement est par exemple maître des horaires/matières de ses élèves. Cette adaptation aux besoins locaux est considérée comme un gage d'efficacité. Et de plus en plus depuis les années 80 , cette autonomie se double d'une régulation ex post impliquant l'État. La politique du New Labour n'instaure à cet égard aucun changement, qui affirme : "I'intervention de l'État dans la vie des écoles sera en proportion inverse de leur succès ". La notion d'efficacité monte en force, et si le gouvernement a cru bon de "reprendre la main » eu égard aux objectifs généraux (le curriculum national et les "standards" qui en découlent), il ne revient pas sur l'autonomie des écoles, se contentant, si l'on peut dire, d'évaluer dans quelle mesure ces dernières atteignent les objectifs fixés. L'État sait également intervenir de manière ferme quand les objectifs ne sont pas atteints (avec mise sous tutelle des écoles considérées comme défaillantes). On assiste donc à la fois à une " centralisation " par le biais du National Curriculum, et au développement d'un pilotage par l'aval (où les "usagers" tiennent une place de plus en plus importante), que l'on peut lire comme une décentralisation croissante (Fialaire, 1995 et 1996).

L'exemple anglais montre bien que l'autonomie des établissements n'implique pas leur abandon dans un marché sauvage ; I'État peut être à la fois modeste (ne suggérant pas les "bonnes formes" pédagogiques) et ferme, voire impitoyable, dans la régulation ex post qu'il exerce. C'est une conception de la décentralisation très différente de l'acception française, qui combine une forte tradition de régulation par les normes, une volonté relativement récente et ambivalente de décentralisation et une certaine crainte par rapport à un pilotage par les résultats.

Pour finir, retenons que parmi les spécificités du contexte britannique, tant dans la manière de penser les inégalités sociales face à l'école que dans l'articulation entre recherche et politiques éducatives, il y a tout d'abord l'importance des débats de fond sur le système éducatif, débats traversés par des oppositions politiques tran- 
chées, et aussi l'insertion des sociologues (du moins de leurs analyses) dans ces débats politiques. Notons également le caractère moins tabou, y compris parmi le milieu des sociologues, du débat autour des différences entres élèves, qu'il s'agisse des inégalités d'aptitudes et même autour de la question de l'existence de "goûts " différents selon les milieux sociaux; par exemple, pour expliquer la moindre scolarisation des enfants de milieu populaire, certains sociologues se risquent à invoquer une "working class indifference » face à l'éducation (Murphy, 1990).

\section{ANALYSER ET INTERPRÉTER LES INÉGALITÉS SOCIALES DE SCOLARISATION}

La comparaison entre la France et la GrandeBretagne est d'emblée stimulante, en ce qu'elle amène à confronter des données de prime abord étonnantes. Partons d'une première approche, très globale, des inégalités sociales globales par rapport à l'éducation. On peut mettre en regard (par des analyses de régression) le plus haut niveau éducatif atteint et un certain nombre de variables caractérisant l'origine sociale de l'individu, ceci par groupes de générations, si on entend caractériser une évolution. Dans ce cas, le pourcentage de la variance du niveau éducatif $\left(R^{2}\right)$ expliquée par ces variables constitue un indicateur simple de la force des inégalités sociales face à l'éducation. II renseigne, en d'autres termes, sur la valeur du pronostic, quant au niveau d'éducation qu'atteindra un individu, que l'on peut faire sur la base de l'information fournie sur son origine sociale.

En Grande-Bretagne, ce type d'analyse débouche sur un résultat de prime abord étonnant, à savoir le niveau relativement faible du $R^{2}$ $(17,7 \%$, contre $26,7 \%$ pour la France sur la même période). Ceci reflète une association moins forte, en Grande-Bretagne, entre caractéristiques familiales et niveau d'instruction atteint. Ce pays est d'ailleurs l'un des pays les moins inégalitaires à cette aune, parmi ceux pour lesquels cet indicateur est disponible (Allemagne, Italie, notamment, deux pays assez proches de la France ; cf. Duru-Bellat et Kieffer, 1999a, 1999b).

Le cas anglais apparaît relativement intriguant pour deux raisons. Tout d'abord parce que, de manière générale, le Royaume-Uni présente un niveau d'inégalité plutôt supérieur aux pays cités (d'après les chiffres de l'OCDE sur les inégalités de revenus). Et aussi parce que les scolarisations s'y sont beaucoup moins développées qu'en France tout au long du $X X^{e}$ siècle (pour ne citer qu'un seul chiffre, dans la génération 70 , l'âge médian de fin d'études - à temps plein - se situe en Grande-Bretagne au même niveau qu'en France pour les cohortes des années 30). Dans la mesure où l'on sait que le niveau d'inégalités face à l'école est d'autant plus fort que les scolarisations sont moins développées, on s'attendrait à ce que les inégalités de carrières scolaires soient plutôt plus prononcées en Grande-Bretagne.

Pour tenter de comprendre cette situation énigmatique, il faut adopter une perspective plus analytique sur les inégalités de carrières scolaires, en distinguant inégalités de réussite et inégalités d'orientation ou, plus largement, de cheminement dans le système. Concernant les inégalités de réussite, nous utiliserons des travaux en cours de Broadfoot et Osborn (1997). On y observe, à l'aune de tests scolaires comparables, une plus forte inégalité des performances des élèves de primaire en fonction de leur niveau social, en langue maternelle et en mathématiques, les performances des élèves de milieu socio-économique défavorisé étant particulièrement faibles en Grande-Bretagne. Ceci s'accompagne d'attitudes moins favorables par rapport à la scolarité : par rapport à leurs homologues français, les élèves de milieu populaire sont plus nombreux à dire qu'ils ne se plaisent pas à l'école, ou encore donnent une place plus faible à l'obtention d'un diplôme dans leurs projets d'avenir. Le vécu scolaire et les représentations de l'enjeu scolaire sont plus uniformes chez les élèves français.

On ne dispose malheureusement pas d'éléments permettant des comparaisons des niveaux de réussite sur une base plus large et à des stades plus avancés du cursus (les données britanniques sont souvent manquantes dans les grandes enquêtes internationales telles que l'enquête de I'IEA « Reading Literacy »). On ne saurait donc conclure, bien que cela reste une possibilité, à des inégalités sociales de réussite plus marquées en Grande-Bretagne.

Mais quel que soit le niveau de réussite, on sait que des inégalités sociales spécifiques affectent 
le déroulement des carrières scolaires. En Grande-Bretagne, l'accès au «secondaire inférieur " a longtemps été très discriminant, nous l'avons évoqué. Les élèves accédaient à 11 ans soit à une école de type sélectif (école privée ou Grammar school publique), soit à une filière non sélective (secondary modern, secondary technical). Ces inégalités sociales sont stables jusqu'aux générations nées au milieu du siècle: même si l'on a rendu gratuites les écoles sélectives en 1944, ceci prend place dans un contexte d'ouverture limitée (30\% des jeunes y accèdent dans les générations 1913-1922, contre $35 \%$ dans les générations 1943-1952). Tout se passe comme si la gratuité n'avait fait qu'accroître la concurrence, puisque dans un contexte d'accroissement de la demande, l'offre est restée relativement stable. On n'est donc pas surpris de voir les inégalités sociales remonter quelque peu, les enfants de milieu aisé étant les premiers à profiter de cette discrète ouverture, sachant qu'on est encore loin des « effets de plafond " qui rendraient la démocratisation mécanique (dans les générations 43-52, les trois quarts des enfants de la "service class » accèdent à une école sélective, tandis que les trois-quarts des enfants de la «working class» accèdent à une secondary modern ou une comprehensive).

Comme en France, la suppression des obstacles matériels ne s'avère donc pas suffisante. Le maintien de structures diversifiées dans le secondaire britannique a joué le même rôle que la permanence de filières après l'instauration du collège en France. Mais la Grande-Bretagne reste néanmoins un peu en avance sur la France: les écoles de type «compréhensif » deviennent majoritaires au cours des années 70 (elles scolarisent $79 \%$ des élèves en 1977, un peu plus de $90 \%$ en 1994), alors que la suppression des filières prévue par la réforme Haby n'intervient qu'en 1978 (et qu'une orientation en fin de 5ème subsiste jusqu'au milieu des années 80). Cela dit, le groupement des élèves par niveau-matière (le «setting ») atténue quelque peu, en Grande-Bretagne, le caractère commun de cette scolarité.

La portée démocratisante de la généralisation des écoles «compréhensives » a été évaluée précisément pour la première fois par Mc Pherson et Willms (1987) et Gamoran (1996), à partir d'enquêtes centrées sur l'Écosse. Sont prises en compte des générations nées entre 1960 et 1968 , avec une évaluation précise des résultats des élèves (au Scottish Certificate of Education) et pas seulement leur accès à un niveau. À cette aune, les performances des jeunes de milieu populaire, notamment des garçons, se sont améliorées, même s'il subsiste des écarts sociaux de performance aux niveaux de réussite les plus élevés. Sur des données encore plus récentes, la recherche de Gamoran conforte ce résultat: on assiste à une élévation des scores moyens, qu'il faut imputer selon l'auteur à une baisse des inégalités entre écoles (et aussi à une évolution vers une standardisation accrue des programmes) ; mais une fois de plus, des inégalités sociales sont observées aux niveaux de réussite les plus élevés (quand il s'agit d'obtenir un «pass» permettant d'entrer dans l'enseignement supérieur).

Les chercheurs attribuent cette baisse des inégalités notamment à la réduction des écarts de niveau scolaire et de ségrégation sociale entre les écoles; la généralisation des écoles «compréhensives " aurait donc homogénéisé le niveau des jeunes, sans parvenir à réduire les inégalités de réussite parmi l'“élite » scolaire. II faut mettre ce constat en perspective avec l'individualisation (le “profilage ») de l'évaluation qui existe en GrandeBretagne : le souci de voir chaque élève, quel que soit son niveau, valider quelque chose au GCSE, nourrit la constitution de groupes de niveaumatière (le «setting»), chaque groupe préparant à tel niveau de difficulté à l'examen. Ceci contrecarre très largement l'unification des curricula que l'on prétend développer par ailleurs et qui était en tout cas au cœur du projet compréhensif. Les racines de la résistance à l'indifférenciation sont en l'occurrence très anciennes, puisant dans cette conviction dans le bien-fondé d'une diversification de l'enseignement en fonction des aptitudes, dont nous avons parlé dans la première partie.

Les développements récents du système anglais vont d'ailleurs dans ce sens, depuis l'Education Act de 1988 : on encourage en effet la concurrence entre écoles et le maintien (voire le léger développement), à côté des comprehensive schools, très majoritaires et non remises en cause, d'alternatives en nombre croissant (les City Technology Colleges, les Grant-Maintained Schools, sans parler des Grammar Schools qui existent encore). II est probable qu'il en résulte une ségrégation scolaire et sociale accrue entre les écoles (cf. Hardman et Levacic, 1999). La 
ségrégation scolaire résulterait de l'«écrémage 》 que réalisent ces écoles qui ont le pouvoir de sélectionner leurs élèves. La ségrégation sociale résulterait à la fois du système de financement des écoles au prorata du nombre d'élèves et des biais sociaux qui marquent les choix. Les sociologues britanniques ont engagé de très nombreux travaux sur cette question, allant d'analyses monographiques de "marchés locaux» et de la manière dont les écoles s'y positionnent, à des enquêtes statistiques sur les choix des familles ou l'évolution du recrutement social et ethnique des écoles du fait de la concurrence.

Et, situation assez typique des pays anglosaxons, des polémiques scientifiques nombreuses émaillent les revues spécialisées quant aux effets de la " mise en marché » (cf. par exemple Gibson et Asthana, 1999, versus Gorard et Fitz, 1998). Ces polémiques sont très instructives pour le lecteur français, dans la mesure où :

1) elles mettent en exergue des points de méthode qui sont, comme c'est souvent le cas, des questions de fond: par exemple, quel est le niveau géographique le plus pertinent pour observer la polarisation, éventuelle, des écoles : l'écheIon administration de base que sont les LEA (Local Education Authorities), ou les «marchés locaux »?

2) elles abordent sans tabou toutes les questions: par exemple, le système de choix, si connoté par l'idéologie libérale qu'il soit, n'est-il pas plutôt moins inégalitaire que le système de la carte scolaire ?... Toujours est-il que cette question reste largement ouverte, mais que l'on est assez sûr d'obtenir à brève échéance des réponses scientifiquement bien documentées, les chercheurs britanniques n'excluant pas de leur responsabilité d'instruire les questions du politique.

Revenons rapidement à ce que l'on sait du déroulement de la suite des carrières scolaires. Une des particularités du système britannique est que les inégalités s'y concentrent sur le choix d'une école secondaire (au moins jusque dans la période récente, où la comprehensivization est totale) ; la poursuite des études se fonde en effet assez largement ensuite sur des critères méritocratiques; l'effet de l'origine sociale baisse au fur et à mesure du déroulement du cursus (ce qui n'est pas le cas en France). Et de fait, si on examine les inégalités à l'entrée dans le " secondaire supérieur ", le niveau de ces inégalités sociales reste à nouveau relativement faible (cet indicateur de chances relatives qu'est le odds ratio est de 7,5 , contre 9 ou un peu plus en Allemagne ou en France). On peut souligner que le développement marqué des formations professionnelles non académiques n'a pas constitué un vecteur de démocratisation car tous les milieux sociaux en ont bénéficié (à la différence de ce que l'on observe en France).

En ce qui concerne l'enseignement supérieur, une caractéristique du système britannique est, au moins jusqu'aux générations prises en compte dans les recherches, son relativement faible développement. Ainsi, parmi les générations nées entre 1956 et 1965, seulement $55 \%$ environ des enfants de la "service class " entrent dans l'enseignement supérieur. Les statistiques plus récentes (portant sur la génération 70 ) montrent que le taux d'étudiants entre 20 et 24 ans est moitié moins élevé en Grande-Bretagne qu'en France (11,4 contre 23,3\%). Le système britannique est par ailleurs fortement hétérogène. Coexistent en effet des Universités, voie la plus sélective socialement, des Polytechnics et des Colleges. Les odds ratios calculés sur les générations 1946-1955 étaient de 12,9 pour les premières, de 3,9 pour les secondes, et de 3,4 pour les Colleges. Les inégalités dans l'obtention d'un diplôme du Supérieur seraient néanmoins en baisse légère en Grande-Bretagne, mais il est probable, bien que nous ne disposions pas de chiffres précis sur ce point, que le niveau des inégalités est devenu plus faible en France, précisément du fait du développement exceptionnel des scolarités dans le Supérieur.

Au total les chercheurs anglais concluent à un déplacement des inégalités : celles-ci porteraient à présent sur la qualification obtenue (au GSE, O level, puis à ce nouvel examen qu'est le GCSE), qui serait devenue "l'équivalent fonctionnel » de l'accès à une scolarité secondaire dans une école sélective, les écarts sociaux ayant peu changé entre les générations nées après 1960 et celles nées au début du siècle. Mais les inégalités sociales auraient, au Royaume-Uni comme en France, changé d'allure (et donc vraisemblablement de sens pour les élèves) : elles ne prennent plus place dès 11 ans, lors de l'accès à un type d'école secondaire, qui auparavant déterminait toute la carrière scolaire ; aujourd'hui, les inégalités subsistent, quel que soit le type d'école fré- 
quentée, dès lors que c'est la réussite au cours de la scolarité secondaire qui est prise en compte, en particulier l'accès aux niveaux les plus élevés de qualification scolaire.

Pour en revenir à l'interprétation des différences quant au niveau des inégalités sociales entre pays, elle est à l'évidence complexe. Elle invite spontanément à prendre en compte le fonctionnement même des systèmes, certes leur structure (unifiée ou non, durée des scolarisations), mais surtout les règles qui régissent le cheminement des élèves et leur mode de certification. On observe à la fois des inégalités précoces de performance plus marquées en Grande-Bretagne, et des carrières scolaires sans doute moins porteuses d'inégalités sociales spécifiques du fait du caractère plus "méritocratique " des cheminements des élèves. Et au total, une relation entre origine sociale et niveau éducatif atteint moins inégalitaire.

Mais le fonctionnement du système scolaire ne saurait tout expliquer. II faut aussi prendre en compte les anticipations par les acteurs de ce qu'ils feront de leur diplôme. Et à cet égard le fait que le diplôme constitue un enjeu plus ou moins fort n'a sans doute pas un caractère accessoire. On conçoit bien que les inégalités sociales face à l'éducation puissent être plus marquées dans les pays où l'insertion est très dépendante du diplôme obtenu (les familles, inégales, mobilisent alors d'autant plus leurs atouts, inégaux). Une des caractéristiques marquantes de la GrandeBretagne (comme des pays voisins comme I'Irlande d'ailleurs) à cet égard est la relative faiblesse des relations entre formation et emploi, par rapport à des pays comme l'Allemagne ou la France, sachant que le lien entre formation et emploi se serait encore affaibli ces dernières années en Grande-Bretagne (Shavit et Müller, 1998, cf. aussi Breen, 1998). Dans ce pays, la formation professionnelle s'effectue couramment sur le tas et non à partir d'une certification nationalement reconnue (même si la situation évolue, cf. Jobert, Marry et Tanguy, 1995). Notons également que la personne en recherche d'emploi sans diplôme est moins stigmatisée que dans les pays où le niveau moyen de formation est plus élevé (notamment la France). Enfin, on sait qu'en Grande-Bretagne les reprises d'études des adultes sont beaucoup plus répandues.

Faut-il alors faire l'hypothèse qu'une des causes expliquant le caractère moins marqué des inégalités sociales de carrières scolaires en Grande-Bretagne est le moindre enjeu social que les études constituent ? Ce serait sans doute prématuré, car c'est bien l'ensemble des relations entre origine sociale, éducation et position sociale atteinte qu'il conviendrait d'analyser. Une conclusion se dégage néanmoins des travaux récents (cf. notamment Breen, 1998), à savoir que dans la transmission du statut social d'une génération à l'autre, l'école jouerait sans doute un rôle moins fort que celui qu'on postule couramment. Faut-il souligner qu'il ne serait pas incongru de se poser cette question en France?

Pour conclure, soulignons l'intérêt et la valeur heuristiques des débats que les sociologues britanniques engagent à partir de leurs travaux empiriques, en particulier, précisément, sur le rôle de l'école dans la genèse et le renouvellement des inégalités à l'école (avec les analyses de sociologues comme Moore, ou Foster et al.). Dans une méta-analyse critique de la production sociologique sur l'école, ils posent chacun à leur manière la question de savoir si les sociologues n'ont pas tendance à surestimer le poids de ce qui se passe en classe. Tout se passe comme s'ils reprenaient à leur compte le postulat selon lequel toutes les inégalités observées au sein de l'école auraient forcément un rôle dans la reproduction globale des inégalités de résultats et de carrières scolaires. De fait, les inégalités de «traitement " (pédagogique) sont considérées comme des conditions nécessaires et suffisantes des inégalités de résultats et de carrières scolaires. C'est de fait la théorie de la reproduction qui forme l'arrière-fond, en générale implicite, de la plupart de ces travaux (qui se condamnent à n'en être alors qu'une illustration). Difficile alors d'échapper à un certain finalisme (I'école est faite "pour ça "...). Dans ce cas, la théorie est "selfconfirming $» . .$.

Pourtant, et c'est la thèse de Moore, certaines évolutions historiques, comme celle des scolarités des filles, montrent que quelle que soit la force de la socialisation scolaire qui vise à en faire des dominés, les individus sont à même de réussir à l'école dès lors qu'ils y voient leur propre intérêt, compte tenu notamment de ce qui les attend sur le marché du travail. Toujours estil qu'on ne peut pas comprendre les inégalités face à l'école sans prendre en compte ce qui se passe hors de l'école. Moore souligne à ce propos que la polarisation des sociologues sur ce 
qui se joue à l'école n'est peut-être qu'un effet de position: enseignants ou anciens enseignants, travaillant souvent pour leurs collègues praticiens, ils sont amenés à considérer comme décisif ce qui est à la portée des acteurs de l'institution, et font de fait plus une sociologie "pour» l'éducation qu'une sociologie «de» l'éducation. À nouveau, cette sociologie de la sociologie de l'éducation n'aurait-elle qu'une pertinence locale?

Marie Duru-Bellat

Université de Bourgogne

IREDU-CNRS
(1) Ce General Certificate of Secondary Education devait concerner tous les élèves de 16 ans, qui, auparavant, avaient le choix entre le Certificate of Secondary Education, de niveau modeste, et le General Certificate of Education, Ordinary level, conçu pour environ le tiers des meilleurs élèves, s'engageant dans des études secondaires longues.

\section{BIBLIOGRAPHIE}

BREEN R. (1998). - The Persistence of Class Origin Inequalities Among School Leavers in the Republic of Ireland, 1984-1993. British Journal of Sociology, vol. 49, $\mathrm{n}^{\circ} 2,275-298$.

BROADFOOT P. (1986). - Assessment Policy and Inequality : the United Kingdom Experience. British Journal of Sociology of Education, vol. $7, \mathrm{n}^{\circ} 2$, p. 205-224.

BROADFOOT P., OSBORN M. et al. (1997). - Comparing like with like. International Association for Educational Assessment, 23rd annual conference, Durban.

DURU-BELLAT M. (1999). - La sociologie des inégalités sociales à l'école, entre engagement et distanciation. In D. Meuret (éd), La justice du système éducatif. Bruxelles: De Boeck, p. 21-36.

DURU-BELLAT M., KIEFFER A. (1999a). - La démocratisation de l'enseignement "revi-sitée »: une mise en perspective historique et internationale. Cahier de I'IREDU-LASMAS-IDL, $\mathrm{n}^{\circ} 60$.

DURU-BELLAT M., KIEFFER A. (1999b). - Evaluer la démocratisation de l'enseignement: la situation française à l'épreuve des comparaisons internationales. Revue Française de Pédagogie, $n^{\circ} 127$, p. $49-62$.

ERIKSON R., JONSSON J. (1996). - Can Education Be Equalized? Londres: Westview Press.

FIALAIRE J. (1995). - Le processus de réforme de l'enseignement en Grande-Bretagne depuis 1988. Savoir, $n^{\circ} 3$, p. 467-484.

FIALAIRE J. (1996). - La centralisation du système éducatif britannique: le marché s'administre. Revue Française d'Administration Publique, $\mathrm{n}^{\circ} 79$, p. 535-543.

FOSTER P., GOMM R., HAMMERSLEY M. (1996). Constructing Educational Inequality. Londres: The Falmer Press.
GAMORAN A. (1996). - Curriculum Standardization and Equality of Opportunity in Scottish Secondary Education : 1984-1990. Sociology of Education, vol. 69, $\mathrm{n}^{\circ} 1$, p. 1-22.

GIBSON A., ASTHANA S. (1999). - Schools, Markets and Equity; access to secondary education in England and Wales, American Educational Research Association Annual Meeting, Montréal, 21/04/99.

GORARD S, FITZ J. (1998). - The More Things Change... The Missing Impact of Marketisation? British Journal of Sociology of Education, vol. 19, $\mathrm{n}^{\circ} 3$, p. 365-375.

GRAY J. (1999). - The fate of Comprehensive reforms in England during the 1990s. In A. Leschinsky, K.U. Mayer (eds), The Comprehensive School Experiment Revisited : Evidence from Western Europe. Berne : Peter Lang.

HARDMAN J., LEVACIC R. (1999). - La concurrence entre les établissements secondaires anglais: dynamisme ou différenciation? Carrefours de I'Education, $\mathrm{n}^{\circ}$ 7, p. 136-161.

HEATH A., JACOBS S. (1999). - Comprehensive Reform in Britain. In A. Leschinsky, K.U. Mayer (eds), The Comprehensive School Experiment Revisited: Evidence from Western Europe, Berne: Peter Lang.

JOBERT A., MARRY C., TANGUY L. (1995). - Education et travail en Grande-Bretagne, Allemagne et Italie. Paris : Colin.

JONSSON J., MILLS C. (1993). - Social Class and Educational Attainment in Historical Perspective: A Swedish-English Comparison. British Journal of Sociology, vol. 44, $n^{\circ} 2$, p. 213-247 (part I) et $n^{\circ} 3$, p. 403-428 (part II).

JONSSON J., MILLS C., MÜLLER W. (1996). - A Half Century of Increasing Educational Openness? Social Class, Gender and Educational Attainment in 
Sweden, Germany and Britain. In Erikson et Jonsson (eds.), op. cit.

Mc PHERSON A., WILLMS J.D. (1987). - Equalisation and Improvement: Some Effects of Comprehensive Reorganisation in Scotland. Sociology, $n^{\circ} 21$, p. 509-539.

MOORE R. (1996). - Back to the Future: The Problem of Change and the Possibilities of Advance in the Sociology of Education. British Journal of Sociology of Education, vol. 17, $\mathrm{n}^{\circ} 2$, p. 145-161.

MÜLLER W., SHAVIT Y. (1998). - The Institutionnal Embeddedness of the Stratification Process. A Comparative Study of Qualifications and Occupa- tions in Thirteen Countries. In Shavit Y., et Müller W. (éds. ), From School to Work. Oxford: Clarendon Press.

MURPHY J. (1990). - A Most Respectable Prejudice: Inequalities in Educational Research and Policy. British Journal of Sociology, vol. 43, $n^{\circ} 1$, p. 2954.

SHAVIT Y., BLOSSFELD H-P. (éds. ) (1993). - Persistent Inequality. Londres: Westview Press.

SHAVIT Y., MÜLLER W. (1998). - From School to Work. Oxford: Clarendon Press.

WALFORD G. (1994). - Choice and Equity in Education. Londres : Cassell. 